\title{
Optical Rotation
}

National Cancer Institute

\section{Source}

National Cancer Institute. Optical Rotation. NCI Thesaurus. Code C103201.

The ability of a chiral substance to rotate the plane of polarization of a beam of transmitted plane-polarized light, either clockwise (to the right) or counterclockwise (to the left). 